\title{
Childhood Malignant Peripheral Nerve Sheath Tumor
}

National Cancer Institute

\section{Source}

National Cancer Institute. Childhood Malignant Peripheral Nerve Sheath Tumor. NCI

Thesaurus. Code C8094.

A malignant peripheral nerve sheath tumor occurring in children. 